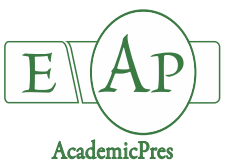

Cebeci E et al. (2020)

Notulae Botanicae Horti Agrobotanici Cluj-Napoca 48(2):604-614

DOI: $10.15835 /$ nbha 48211932

Research Article

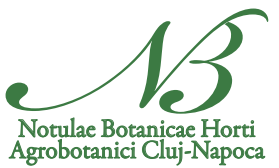

\title{
Morphologic and molecular assessments of cucumber (Cucumis sativus L.) landraces
}

\author{
Esra CEBECI*, Volkan GOZEN, Levent KESKIN, \\ Aytul YILDIRIM
}

\author{
Bati Akdeniz Agricultural Research Institute-07100,Muratpasa, Antalya,Turkey;volkan.gozen@tarimorman.gov.tr; \\ levent.keskin@tarimorman.gov.tr; aytul.kitapci@tarimorman.gov.tr; \\ esrac3@hotmail.com (*correspondingauthor)
}

\begin{abstract}
In this study, 90 locally grown cucumber (Cucumis sativus L.) landraces were collected and morphologically characterized using 20 descriptors derived from UPOV (International Union for the Protection of New Varieties of Plants). Genetic diversity and relationships of the genotypes were revealed using 20 sequence-related amplified polymorphism (SRAP) marker combinations. The discrimination power of each polymorphic marker (estimated by the polymorphism information content) ranged from 0.15 to 0.99 with an average of 0.73 . Dice's similarity coefficient ranged between $0.00-1.00$. The cluster analysis that was conducted using the unweighted pair group method of arithmetic averages (UPGMA) for both molecular and morphologic data showed that all of the genotypes fell into two main groups and many subdivisions. According to morphological data, fruit length, diameter and weight of the genotypes were determined between $6.5-32.5$ $\mathrm{cm}, 25-52 \mathrm{~mm}$ and, $28-625 \mathrm{~g}$ respectively. It is clear from the results, a moderate level of genetic diversity, which has the potential for broadening the genetic base, was observed among the Turkish cucumber landraces.
\end{abstract}

Keywords: breeding; characterization; collection; diversity; NTSYS; SRAP

\section{Introduction}

Cucumber (Cucumis sativus L.) is grown widely in open field or glasshouse conditions. It has modest levels of vitamin A, C, and, some phenolic compounds known to have antioxidant properties (Kumar et al., 2010). India is the primary center of diversity for cucumber (Wehner and Robinson, 1991). It has a narrow genetic base with a genetic variability of $3-8 \%$ so it is more susceptible to biotic and abiotic stresses (Rao and Hodgkin, 2002).

Nowadays, climate change threatens to reduce crop yield and harming the food security. Local landraces have adaptation skills to shifting climatic conditions. They have been a major resource for crop breeding, due to their tolerance to the extreme climate changes and the resistance to some pests and diseases (Rao and Hodgkin, 2002). Their genetic and phenotypic features need to be characterized carefully for the proper use in breeding programs (Parisi et al., 2017). The success of any plant breeding program depends on the genetic 
variability (Bartaula et al., 2019) Establishing a gene pool and determination of their relationships is the first step for plant improvement (Normohammadi et al., 2017).

Morphological markers are good determinants but they are not stable and they influenced by ecologic conditions (Fares et al., 2009). Molecular markers are not affected by the environmental changes and they serve as a tool to overcome the deficiencies of morphological markers in the characterization studies (Agarwal et al., 2008; Gostimsky et al., 2005; Gulsen and Mutlu, 2005). For this reason, using morphological and molecular marker systems together is preferred for the better clarification of the relationships between the genotypes.

In this research, 20 descriptors which were derived from "International Union for Protection of New Plant Varieties" (UPOV) were used for the morphological determinations and the most polymorphic 20 SRAP marker combinations were used for the molecular characterization. SRAP is a PCR-based technique. It depends on the amplification of open reading frames (ORFs) and two-primer amplifications. The primer sequences are random and they have 17-21 nucleotides in length (Agarwal et al., 2008).

Turkey is located on the transition zones. Its geographic position and the variety of agro-climatic conditions caused great biodiversity. This research aimed to establish a characterized gene pool formed by cucumber landraces cultivated in different regions of Turkey. Even though many studies on Turkish cucumber diversity have been completed earlier (Gozen, 2008; Aydemir, 2009), for the first time with this research Turkish landraces were collected and characterized using morphologic and molecular markers together. Therefore, obtained results are significant both for the local and international researchers.

\section{Materials and Methods}

\section{Material}

This study was conducted at Bati Akdeniz Agricultural Research Institute-Antalya located between $36^{\circ} 55^{\prime} 0.98^{\prime \prime} \mathrm{N} 31^{\circ} 05^{\prime} 56.00^{\prime \prime} \mathrm{E}$ coordinates. Ninety landraces were collected from 52 different areas, used as plant material. The information about their given codes, cultivation areas, and geographic locations were presented in Table 1. Thirty-six accessions came from the Genebank of Aegean Agricultural Research Institute and the other 54 accessions collected from cucumber cultivation areas via agricultural district offices before starting to this study.

\section{Methods}

Gene pool establishment

Collected genotypes were selfed for two generations to avoid risks because of possible seed mixture. For this reason, all seeds were planted in 2017 spring and autumn season. When the seedlings at the 2-3 leaf stage, they were transplanted to the greenhouse. During the vegetation period, watering, fertilizing, and diseases and pest management were carefully followed. At the fruiting stage, mixed accessions were detected; they were considered as another genotype and coded differentially. Pollination studies were made in each population separately. Hand pollinated flowers were tagged with coloured materials. Following pollination, after 50-55 days, ripen fruits were harvested; seeds were washed for cleaning and dried then kept in cooled storage until the next season.

\section{Morphological characterization}

Spring and autumn period of 2018, morphological characterization of the genotypes was carried out according to the descriptors were given in Table 2. Five plants per accession were characterized, with 20 descriptors, eight of them referred to plant traits and 12 related to the fruit. 
Cebeci E et al. (2020). Not Bot Horti Agrobo 48(2):604-614

Table 1. The given codes, their cultivation areas, and geographic locations

\begin{tabular}{|c|c|c|c|c|c|}
\hline Code & $\begin{array}{c}\text { Cultivation } \\
\text { area }\end{array}$ & Geographic location & Code & $\begin{array}{c}\text { Cultivation } \\
\text { area }\end{array}$ & Geographic location \\
\hline YG-2 & Çanakkale & $40^{\circ} 08^{\prime} 60.00^{\prime \prime} \mathrm{N}$ & YG-37 & Osmaniye & $37^{\circ} 04^{\prime} 27.01^{\prime \prime} \mathrm{N}$ \\
\hline YG-4 & Bursa & $40^{\circ} 11^{\prime} 44.12^{\prime \prime} \mathrm{N}$ & YG-38 & Konya & $38^{\circ} 00^{\prime} 28.80^{\prime \prime} \mathrm{N}$ \\
\hline YG-6/1 & Edirne & $41^{\circ} 40^{\prime} 37.88^{\prime \prime} \mathrm{N}$ & YG-39 & Şanlıurfa & $37^{\circ} 08^{\prime} 60.00^{\prime \prime} \mathrm{N}$ \\
\hline YG-6/2 & Edirne & $41^{\circ} 40^{\prime} 37.88^{\prime \prime} \mathrm{N}$ & YG-40 & Hatay & $36^{\circ} 25^{\prime} 29.39^{\prime \prime} \mathrm{N}$ \\
\hline YG-6/3 & Edirne & $41^{\circ} 40^{\prime} 37.88^{\prime \prime} \mathrm{N}$ & YG-42 & Amasya & $40^{\circ} 39^{\prime} 11.99^{\prime \prime} \mathrm{N}$ \\
\hline YG-6/4 & Edirne & $41^{\circ} 40^{\prime} 37.88^{\prime \prime} \mathrm{N}$ & YG-44 & Manisa & $38^{\circ} 36^{\prime} 43.27^{\prime \prime} \mathrm{N}$ \\
\hline YG-7 & Zonguldak & $41^{\circ} 26^{\prime} 59.99^{\prime \prime} \mathrm{N}$ & YG-45 & Çankırı & $40^{\circ} 35^{\prime} 59.82^{\prime \prime} \mathrm{N}$ \\
\hline YG-7/2 & Zonguldak & $41^{\circ} 26^{\prime} 59.99^{\prime \prime} \mathrm{N}$ & YG-46 & Çorum & $40^{\circ} 32^{\prime} 56.00^{\prime \prime} \mathrm{N}$ \\
\hline YG-8 & Kocaeli & $40^{\circ} 51^{\prime} 7.20^{\prime \prime} \mathrm{N} 29^{\circ} 52^{\prime} 40.80^{\prime \prime}$ & YG-47 & Niğde & $37^{\circ} 57^{\prime} 56.99^{\prime \prime} \mathrm{N}$ \\
\hline YG-12 & Tokat & $40^{\circ} 18^{\prime} 50.00^{\prime \prime} \mathrm{N}$ & YG-48 & Gaziantep & $37^{\circ} 03^{\prime} 33.98^{\prime \prime} \mathrm{N}$ \\
\hline YG-12/1 & Tokat & $40^{\circ} 18^{\prime} 50.00^{\prime \prime} \mathrm{N}$ & YG-49 & Erzincan & $39^{\circ} 44^{\prime} 59.99^{\prime \prime} \mathrm{N}$ \\
\hline YG-13 & Trabzon & $41^{\circ} 00^{\prime} 18.00^{\prime \prime} \mathrm{N}$ & YG-50 & Kayseri & $38^{\circ} 37^{\prime} 17.99^{\prime \prime} \mathrm{N}$ \\
\hline YG-14 & Afyon & $38^{\circ} 45^{\prime} 24.01^{\prime \prime} \mathrm{N}$ & YG-51 & Samsun & $41^{\circ} 17^{\prime} 15.00^{\prime \prime} \mathrm{N} 36^{\circ} 20^{\prime} 0.60^{\prime \prime}$ \\
\hline YG-14/2 & Afyon & $38^{\circ} 45^{\prime} 24.01^{\prime \prime} \mathrm{N}$ & YG-52 & Gaziantep & $37^{\circ} 03^{\prime} 33.98^{\prime \prime} \mathrm{N}$ \\
\hline YG-15 & Rize & $40^{\circ} 55^{\prime} 32.39^{\prime \prime} \mathrm{N}$ & YG-53 & Hatay & $36^{\circ} 25^{\prime} 29.39^{\prime \prime} \mathrm{N}$ \\
\hline YG-16/1 & Amasya & $40^{\circ} 39^{\prime} 11.99^{\prime \prime} \mathrm{N}$ & YG-54 & Ankara & $39^{\circ} 55^{\prime} 11.53^{\prime \prime} \mathrm{N}$ \\
\hline YG-16/2 & Amasya & $40^{\circ} 39^{\prime} 11.99^{\prime \prime} \mathrm{N}$ & YG-55 & Kayseri & $38^{\circ} 37^{\prime} 17.99^{\prime \prime} \mathrm{N}$ \\
\hline YG-17 & Kütahya & $39^{\circ} 25^{\prime} 27.01^{\prime \prime} \mathrm{N}$ & YG-56 & Hatay & $36^{\circ} 25^{\prime} 29.39^{\prime \prime} \mathrm{N}$ \\
\hline YG-17/2 & Kütahya & $39^{\circ} 25^{\prime} 27.01^{\prime \prime} \mathrm{N}$ & YG-57 & Zonguldak & $41^{\circ} 26^{\prime} 59.99^{\prime \prime} \mathrm{N}$ \\
\hline YG-18 & Bilecik & $40^{\circ} 08^{\prime} 30.91^{\prime \prime} \mathrm{N}$ & YG-58 & Karaman & $37^{\circ} 10^{\prime} 31.20^{\prime \prime} \mathrm{N}$ \\
\hline YG-19 & Trabzon & $41^{\circ} 00^{\prime} 18.00^{\prime \prime} \mathrm{N}$ & YG-59 & Konya & $38^{\circ} 00^{\prime} 28.80^{\prime \prime} \mathrm{N}$ \\
\hline YG-20 & Gümüşhane & $40^{\circ} 27^{\prime} 22.19^{\prime \prime} \mathrm{N}$ & YG-60 & Mersin & $36^{\circ} 47^{\prime} 42.94^{\prime \prime} \mathrm{N} 34^{\circ} 37^{\prime} 4.51^{\prime \prime}$ \\
\hline YG-21 & Aydın & $37^{\circ} 50^{\prime} 39.84^{\prime \prime} \mathrm{N}$ & YG-61 & Mersin & $36^{\circ} 47^{\prime} 42.94^{\prime \prime} \mathrm{N} 34^{\circ} 37^{\prime} 4.51^{\prime \prime}$ \\
\hline YG-22 & Artvin & $41^{\circ} 07^{\prime} 60.00^{\prime \prime} \mathrm{N}$ & YG-64 & Aksaray & $38^{\circ} 22^{\prime} 21.18^{\prime \prime} \mathrm{N}$ \\
\hline YG-22/2 & Artvin & $41^{\circ} 07^{\prime} 60.00^{\prime \prime} \mathrm{N}$ & YG-65 & Mardin & $37^{\circ} 18^{\prime} 47.12^{\prime \prime} \mathrm{N}$ \\
\hline YG-23 & Muğla & $37^{\circ} 13^{\prime} 5.05^{\prime \prime}$ N $28^{\circ} 21^{\prime} 59.40^{\prime \prime}$ & YG-66 & Șanlıurfa & $37^{\circ} 08^{\prime} 60.00^{\prime \prime} \mathrm{N}$ \\
\hline YG-23/2 & Muğla & $37^{\circ} 13^{\prime} 5.05^{\prime \prime}$ N $28^{\circ} 21^{\prime} 59.40^{\prime \prime}$ & YG-67 & Antalya & $36^{\circ} 54^{\prime} 29.23^{\prime \prime} \mathrm{N}$ \\
\hline YG-24 & Mus & $38^{\circ} 43^{\prime} 53.87^{\prime \prime} \mathrm{N} 41^{\circ} 29^{\prime} 5.35^{\prime \prime}$ & YG-68 & Diyarbakır & $37^{\circ} 54^{\prime} 49.07^{\prime \prime} \mathrm{N} 40^{\circ} 13^{\prime} 1.96^{\prime \prime}$ \\
\hline YG-25 & Hakkari & $37^{\circ} 34^{\prime} 27.98^{\prime \prime} \mathrm{N}$ & YG-69 & K.Maras & $37^{\circ} 34^{\prime} 59.99^{\prime \prime} \mathrm{N}$ \\
\hline YG-26 & Kırıkkale & $39^{\circ} 50^{\prime} 59.99^{\prime \prime} \mathrm{N}$ & YG-70 & Manisa & $38^{\circ} 36^{\prime} 43.27^{\prime \prime} \mathrm{N}$ \\
\hline YG-27 & Kırşehir & $39^{\circ} 08^{\prime} 60.00^{\prime \prime} \mathrm{N}$ & YG-71- & Adana & $37^{\circ} 00^{\prime} 6.01^{\prime \prime} \mathrm{N} 35^{\circ} 19^{\prime} 44.00^{\prime \prime}$ \\
\hline YG-28 & Balıkesir & $39^{\circ} 23^{\prime} 6.00^{\prime \prime} \mathrm{N} 27^{\circ} 31^{\prime} 58.80^{\prime \prime}$ & YG-71- & Adana & $37^{\circ} 00^{\prime} 6.01^{\prime \prime} \mathrm{N} 35^{\circ} 19^{\prime} 44.00^{\prime \prime}$ \\
\hline YG-28/2 & Balıkesir & $39^{\circ} 23^{\prime} 6.00^{\prime \prime} \mathrm{N} 27^{\circ} 31^{\prime} 58.80^{\prime \prime}$ & YG-72 & Antalya & $36^{\circ} 54^{\prime} 29.23^{\prime \prime} \mathrm{N}$ \\
\hline YG-29 & Kastamonu & $41^{\circ} 22^{\prime} 11.99^{\prime \prime} \mathrm{N} 33^{\circ} 46^{\prime} 9.59^{\prime \prime}$ & YG-74 & Erzincan & $39^{\circ} 44^{\prime} 59.99^{\prime \prime} \mathrm{N}$ \\
\hline YG-30 & İzmir & $38^{\circ} 25^{\prime} 19.20^{\prime \prime} \mathrm{N}$ & YG-77 & Yozgat & $39^{\circ} 49^{\prime} 12.00^{\prime \prime} \mathrm{N}$ \\
\hline YG-31 & Sivas & $39^{\circ} 31^{\prime} 6.59^{\prime \prime} \mathrm{N} 37^{\circ} 17^{\prime} 25.20^{\prime \prime}$ & YG-78 & Bursa & $40^{\circ} 11^{\prime} 44.12^{\prime \prime} \mathrm{N}$ \\
\hline$Y G-31 / 2$ & Sivas & $39^{\circ} 31^{\prime} 6.59^{\prime \prime} \mathrm{N} 37^{\circ} 17^{\prime} 25.20^{\prime \prime}$ & YG- & Bursa & $40^{\circ} 11^{\prime} 44.12^{\prime \prime} \mathrm{N}$ \\
\hline YG-32 & Yozgat & $39^{\circ} 49^{\prime} 12.00^{\prime \prime} \mathrm{N}$ & YG-79 & Karaman & $37^{\circ} 10^{\prime} 31.20^{\prime \prime} \mathrm{N}$ \\
\hline YG-32/2 & Yozgat & $39^{\circ} 49^{\prime} 12.00^{\prime \prime} \mathrm{N}$ & YG-81 & Mersin & $36^{\circ} 47^{\prime} 42.94^{\prime \prime} \mathrm{N} 34^{\circ} 37^{\prime} 4.51^{\prime \prime}$ \\
\hline YG-33 & Nevșehir & $38^{\circ} 37^{\prime} 30.00^{\prime \prime} \mathrm{N}$ & YG-82 & Antalya & $36^{\circ} 54^{\prime} 29.23^{\prime \prime} \mathrm{N}$ \\
\hline YG-33/2 & Nevsehir & $38^{\circ} 37^{\prime} 30.00^{\prime \prime} \mathrm{N}$ & YG-83 & Kilis & $36^{\circ} 42^{\prime} 57.96^{\prime \prime} \mathrm{N}$ \\
\hline YG-34 & Çanakkale & $40^{\circ} 08^{\prime} 60.00^{\prime \prime} \mathrm{N}$ & YG-85 & Osmaniye & $37^{\circ} 04^{\prime} 27.01^{\prime \prime} \mathrm{N}$ \\
\hline YG-35 & İstanbul & $41^{\circ} 00^{\prime} 49.82^{\prime \prime} \mathrm{N}$ & YG-89 & Malatya & $38^{\circ} 21^{\prime} 0.65^{\prime \prime} \mathrm{N} 38^{\circ} 19^{\prime} 0.01^{\prime \prime} \mathrm{E}$ \\
\hline YG-36 & Samsun & $41^{\circ} 17^{\prime} 15.00^{\prime \prime} \mathrm{N} 36^{\circ} 20^{\prime} 0.60^{\prime \prime}$ & YG-90 & Adana & $37^{\circ} 00^{\prime} 6.01^{\prime \prime} \mathrm{N} 35^{\circ} 19^{\prime} 44.00^{\prime \prime}$ \\
\hline YG-36/2 & Samsun & $41^{\circ} 17^{\prime} 15.00^{\prime \prime} \mathrm{N} 36^{\circ} 20^{\prime} 0.60^{\prime \prime}$ & YG-91 & Yalova & $40^{\circ} 39^{\prime} 18.04^{\prime \prime} \mathrm{N}$ \\
\hline
\end{tabular}

DNA extraction

Selfed seeds of 90 genotypes were sown again in the spring of 2019 . When the seedlings were at 2 - 4 leaf stage, plant samples were taken to perform DNA extraction. The DNAs of plants were isolated according to the modified CTAB protocol by Doyle and Doyle (1990). After the extraction, the DNA concentrations were determined in $1 \%$ agarose gel. All extracted genomic DNAs were stored at $-20{ }^{\circ} \mathrm{C}$ in TE buffer for SRAP analysis. 
Table 2. Morphologic descriptors derived from UPOV by Gozen (2008)

\begin{tabular}{|c|c|c|c|}
\hline Features & Explanations & Features & Explanations \\
\hline Growth habit & (1) Weak, (3) Medium, (5) Strong & $\begin{array}{l}\text { Intensity of skin } \\
\text { colour }\end{array}$ & (1) Light, (3) Medium, (5) Intense \\
\hline $\begin{array}{l}\text { Length of first } 15 \\
\text { internodes }\end{array}$ & $\begin{array}{l}\text { (1) Very short, (3) Short, (5) } \\
\text { Medium, (7) Long }\end{array}$ & Prickliness on fruit & (1) Absent, (3) Present \\
\hline $\begin{array}{l}\text { Length of } \\
\text { internodes }\end{array}$ & (1) Short, (3) Medium, (5) Long & Colour of prick & (1) Black, (3) White \\
\hline Size of blade & (1) Small, (3) Medium, (5) Big & Groove on fruit & (1) Absent, (3) Medium, (5) \\
\hline $\begin{array}{c}\text { Intensity of green } \\
\text { colnur }\end{array}$ & (1) Light, (3) Medium, (5) Very & Length of peduncle & (1) Short, (3) Medium, (5) Long \\
\hline Sex expression & $\begin{array}{c}\text { (1) Monocie, (3) Gynocie, (5) } \\
\text { Andromonocie }\end{array}$ & $\begin{array}{c}\text { Fruit netting } \\
\text { distribution at seed } \\
\text { harvest stage }\end{array}$ & (1) Absent, (3) Present \\
\hline $\begin{array}{l}\text { Number of female } \\
\text { flowers per node }\end{array}$ & (1) $1,(3) 1 / 3,(5)$ More than 3 & $\begin{array}{l}\text { Fruit skin colour at } \\
\text { physiological ripening }\end{array}$ & $\begin{array}{c}\text { (1) Yellow, (3) Orange (5) Brown, } \\
\text { (7) White, (9) Yellowish-green, } \\
\text { (11) Green }\end{array}$ \\
\hline Parthenocarphy & (1) Absent, (3) present & Average fruit weight & (1) Light, (3) Medium, (5) Heavy \\
\hline Fruit Length & $\begin{array}{l}\text { (1) Short, (3) Medium, (5) Long, } \\
\text { (7) Very long }\end{array}$ & Flowering time & (1) Early, (3) Medium, (5) Late \\
\hline Fruit diameter & $\begin{array}{c}\text { (1) Narrow, (3) Medium, (5) } \\
\text { Broad }\end{array}$ & $\begin{array}{l}\text { Number of lateral } \\
\text { shoots in first } 15 \\
\text { nodes }\end{array}$ & $\begin{array}{c}\text { (1) Few or absent, (3) Medium, (5) } \\
\text { Very }\end{array}$ \\
\hline
\end{tabular}

\section{$\underline{\text { SRAP analysis }}$}

The most polymorphic 20 SRAP primer combinations were determined first before starting this stage. The primers were obtained from the previous studies on cucumber conducted by Ferriol et al. $(2003 ; 2004)$. SRAP primers and their sequences that were used to create 20 combinations were presented in Table 3 . The PCR reaction solution was composed of $1 \mu \mathrm{l}$ DNA (20 ng DNA), $15 \mu \mathrm{l} 1 \mathrm{x}$ PCR master mix, $1 \mu$ l each primer $\left(0.3 \mu \mathrm{M}\right.$ each primer) and $7 \mu \mathrm{lddH} 2 \mathrm{O}$. PCR amplification was 5 minutes for initial denaturation at $94^{\circ} \mathrm{C}$, then 5 cycles composed of denaturing at $94^{\circ} \mathrm{C}$ for 1 minute, annealing at $35^{\circ} \mathrm{C}$ for 1 minute, extension at 72 ${ }^{\circ} \mathrm{C}$ for 1 minute and followed by 35 cycles; heating at $94^{\circ} \mathrm{C}$ for 1 minute, annealing at $55^{\circ} \mathrm{C}$ for 1 minute, extension at $72{ }^{\circ} \mathrm{C}$ for 1 minute and 10 minutes for the final extension at $72{ }^{\circ} \mathrm{C}$. PCR amplifications were repeated twice for each primer combination.

The PCR products were fractionated on 2.5\% high-resolution agarose gel in 1 XTAE buffer at $110 \mathrm{~V}$ for 3 hours and photographed (Kodak Gel Logic 200) under UV light for further analysis. A 100 bp DNA ladder was used as a molecular standard.

\section{Data analysis}

Genetic similarity and phylogenetic relationships were analysed by the UPGMA (Un-weighted pairgroup method, arithmetic average) clustering procedure using the software NTSYS (Numerical Taxonomy Multivariate Analysis System) version pc 2.2 (Rohlf, 1998). For morphological data, each genotype was characterized with descriptors were shown in Table 2. For molecular analysis data, each genotype was identified for each primer based on the presence ( 1 ) and absence ( 0 ) of bands. Similarity indices were calculated according to Dice (1945). Polymorphism rates $(\operatorname{Pr})=$ (Polymorphic allel / Total allel) X 100 and polymorphism information content (PIC) $=1-\Sigma$ fi2 (Smith et al., 1997) were calculated. 
Table 3. SRAP primers and their sequences which were used to create combinations

\begin{tabular}{|c|c|c|c|}
\hline Primers & Sequences & Primers & Sequences \\
\hline Me1 & TGA GTC CAA ACC GGA TA & Em2 & GAC TGC GTA CGA ATT TGC \\
\hline Me2 & TGA GTC CAA ACC GGA GC & Em4 & GAC TGC GTA CGA ATT TGA \\
\hline Me3 & TGA GTC CAA ACC GGA AT & Em5 & GAC TGC GTA CGA ATT AA \\
\hline Me 5 & TGA GTC CAA ACC GGA CC & Em6 & GAC TGC GTA CGA ATT GCA \\
\hline Me6 & TGA GTC CAA ACC GGA AG & Em7 & GAC TGC GTA CGA ATT CAA \\
\hline Me8 & TGA GTC CAA ACC GGA CT & Em10 & GAC TGC GTA CGA ATT CAT \\
\hline Me9 & TGA GTC CAA ACC GGA GG & Em11 & GAC TGC GTA CGA ATT CTA \\
\hline Me10 & TGA GTC CAA ACC GGA AA & Em13 & GAC TGC GTA CGA ATT CTG \\
\hline Me11 & TGA GTC CAA ACC GGA AC & Em14 & GAC TGC GTA CGA ATT CTT \\
\hline Me12 & TGA GTC CAA ACC GGA GA & Em15 & GAC TGC GTA CGA ATT GAT \\
\hline Me15 & CGA ATC TTA GCC GGA TA & Em16 & GAC TGC GTA CGA ATT GTC \\
\hline
\end{tabular}

\section{Results and Discussion}

In this study collected local cucumber genotypes were determined morphologically different. Morphological observations of 47 genotypes using 10 UPOV descriptors were presented in Table 4 as an example. According to this data, genotypes were classified in terms of their growth habit, 28 of them (31\%) determined as strong, 49 of them (54\%) were medium and 13 of them (14\%) were reported as weak. Poor growth leads to plants susceptible to diseases, pests, and harsh environmental conditions. Therefore, while a breeding program set up, plant growth habits should be considered. Seventy-eight of used genotypes were detected as monoecious, regarding the flowering time, lateness was observed on 54 genotypes and 19 of them have no prickliness.

Although cultivated cucumbers are mostly similar in terms of root and stem development, when the fruit characteristics, especially fruit shape is considered they could be quite diverse (Bisognin, 2002). In the current study, fruit length, diameter, and weight of the genotypes were determined between $6.5-32.5 \mathrm{~cm}, 25$ $52 \mathrm{~mm}$, and 28- $625 \mathrm{~g}$ respectively. Features such as fruit length, diameter, and color are used to determine the economic importance of a variety (Kennard and Havey, 1995; Ahmed et al., 2004).

It was understood from the study, while the cucumber is native to India where its wild forms still exist (Sebastian et al., 2010), examined landraces are morphologically quite diverse because of Turkey's location between the transition zones (Figure 1). Genotypes were also observed regarding their number of lateral shoots in the first 15 nodes. While 11 genotypes have no lateral shoots, 79 genotypes have lateral shoots between 4 and 14. In a study, Wehner and Guner (2002) reported that, some genotypes achieve high yielding with branching ability while others reach it with more nodes. Also, the higher yield of some genotypes in their study was associated with both extra branches and nodes.

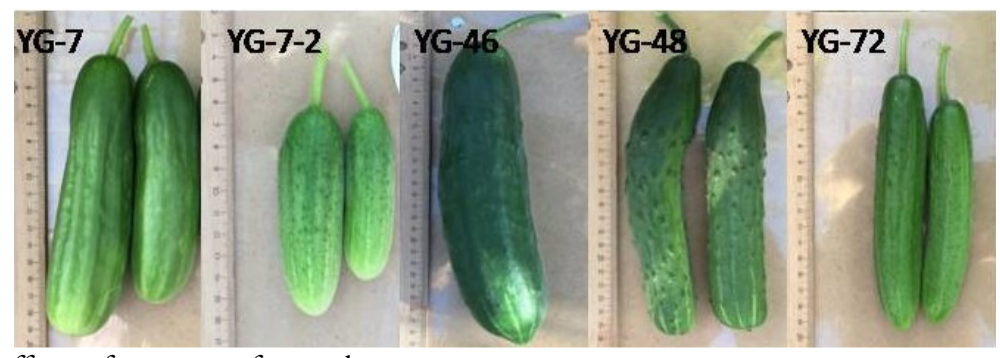

Figure 1. Different fruit types of germplasm 
Table 4. Some of the phenotypic data related to important variable traits of first 47 accessions (remaining 43 accessions' data wasn't presented here to keep the article shorter)

\begin{tabular}{|c|c|c|c|c|c|c|c|c|c|c|}
\hline Genotype & $\begin{array}{c}\text { Growth } \\
\text { habit }\end{array}$ & $\begin{array}{l}\text { Length of } \\
\text { first } 15 \\
\text { inter nodes } \\
(\mathrm{cm})\end{array}$ & $\begin{array}{l}\text { Length of } \\
\text { internode } \\
\quad(\mathrm{cm})\end{array}$ & $\begin{array}{l}\text { Size of } \\
\text { blade } \\
(\mathrm{cm})\end{array}$ & $\begin{array}{c}\text { Number } \\
\text { of female } \\
\text { flowers } \\
\text { per node }\end{array}$ & $\begin{array}{c}\text { Fruit } \\
\text { Length } \\
(\mathrm{cm})\end{array}$ & $\begin{array}{c}\text { Fruit } \\
\text { Diameter } \\
(\mathrm{mm})\end{array}$ & $\begin{array}{c}\text { Averag } \\
\text { e fruit } \\
\text { weight } \\
\text { (g) }\end{array}$ & $\begin{array}{c}\text { Number } \\
\text { of lateral } \\
\text { shoots in } \\
\text { first } 15 \\
\text { nodes }\end{array}$ & $\begin{array}{c}\text { Intensity } \\
\text { of skin } \\
\text { colour }\end{array}$ \\
\hline YG-2 & Medium & 92,7 & 9,7 & 32,0 & 1 & 14,7 & 37,9 & 242 & 11,3 & Medium \\
\hline $\mathrm{YG}-4$ & Medium & 80,0 & 6,3 & 18,6 & 1 & 14,6 & 39,2 & 185,5 & 5,6 & Medium \\
\hline YG-6/1 & Medium & 93,0 & 6,2 & 20,3 & 1 & 6,5 & 53,5 & 230,0 & 6,5 & Light \\
\hline YG-6/2 & Medium & 95,0 & 6,5 & 23,0 & 1 & 6,5 & 55,8 & 250,0 & 8,5 & Medium \\
\hline YG-6/3 & Medium & 93,5 & 6,0 & 22,6 & 1 & 6,5 & 54,9 & 200,0 & 8,5 & Medium \\
\hline YG-6/4 & Strong & 94,5 & 6,1 & 24,3 & 1 & 7,8 & 52,7 & 222,0 & 10,0 & Light \\
\hline YG-7 & Medium & 72,0 & 7,7 & 30,6 & 1 & 14,7 & 40,8 & 165,5 & 7,0 & Medium \\
\hline YG-7/2 & Weak & 72,0 & 7,1 & 24,3 & 1 & 8,3 & 32,8 & 200,0 & 9,5 & Medium \\
\hline YG-8 & Medium & 89,7 & 6,6 & 26,3 & 1 & 14,8 & 39,5 & 91,6 & 11,0 & Light \\
\hline YG-12 & Medium & 105,2 & 8,1 & 25,5 & 1 & 12,3 & 38,2 & 152,0 & 11,5 & Medium \\
\hline YG-12/2 & Medium & 100,2 & 8,0 & 23,5 & 1 & 10,3 & 38,2 & 132,0 & 11,5 & Medium \\
\hline YG-13 & Medium & 94,0 & 8,6 & 29,6 & 1 & 17,1 & 39,35 & 88,0 & 14,0 & Light \\
\hline YG-14 & Weak & 89,0 & 7,2 & 27,0 & 1 & 13,6 & 38,5 & 121,0 & 11,0 & Medium \\
\hline YG-14/2 & Weak & 84,0 & 8,3 & 25,0 & $1-3$ & 13,3 & 36,7 & 237,0 & 9,5 & Medium \\
\hline YG-15 & Weak & 105,0 & 10,6 & 19,0 & $1-3$ & 18,0 & 39,2 & 223,0 & 9,0 & Light \\
\hline YG-16 & Medium & 95,0 & 8,3 & 26,3 & $1-3$ & 12,6 & 30,5 & 123,0 & 6,5 & Medium \\
\hline YG-16/2 & Weak & 112,5 & 7,7 & 29,3 & 1 & 15,8 & 34,5 & 191,0 & 10,0 & Light \\
\hline YG-17 & Medium & 101,0 & 9,1 & 28,0 & $1-3$ & 13,1 & 35,6 & 135,5 & 12,5 & Medium \\
\hline YG-17/2 & Medium & 98,0 & 9,7 & 26,3 & $1-3$ & 15,0 & 37,5 & 185,0 & 12,5 & Medium \\
\hline YG-18 & Weak & 85,0 & 7,7 & 24,6 & $1-3$ & 10,5 & 34,2 & 172,0 & 12,5 & Medium \\
\hline YG-19 & Medium & 83,0 & 8,8 & 28,6 & $1-3$ & 10,1 & 34,5 & 49,0 & 7,5 & Light \\
\hline YG-20 & Strong & 112,5 & 9,8 & 26,6 & $1-3$ & 14,0 & 34,7 & 99,3 & 10,5 & Medium \\
\hline YG-21 & Weak & 94,0 & 8,8 & 27,6 & 1 & 32,5 & 42,05 & 585,0 & 9,5 & Light \\
\hline YG-22 & Strong & 102,5 & 9,6 & 29,3 & $1-3$ & 22,1 & 37,15 & 407,5 & 4,0 & Light \\
\hline YG-22/2 & Medium & 71,0 & 6,2 & 31,0 & 1 & 29 & 37,6 & 560,0 & 6,5 & Medium \\
\hline YG-23 & Medium & 80,5 & 8,2 & 27,6 & $1-3$ & 11 & 35,6 & 183,0 & 10,5 & Light \\
\hline YG-23/2 & Medium & 94,5 & 7,2 & 31,3 & 1 & 13,6 & 35,35 & 54,6 & 11,0 & Light \\
\hline YG-24 & Strong & 98,0 & 8,0 & 31,6 & $1-3$ & 12,6 & 34,95 & 119,0 & 11,5 & Medium \\
\hline YG-25 & Medium & 106,5 & 8,0 & 28,3 & $1-3$ & 9,8 & 38,4 & 109,0 & 12,5 & Intense \\
\hline YG-26 & Strong & 89,5 & 10,0 & 29,3 & $1-3$ & 18 & 39,5 & 28,0 & 11,5 & Light \\
\hline YG-27 & Strong & 97,0 & 9,25 & 28,0 & $1-3$ & 9 & 30,2 & 109,0 & 10,5 & Light \\
\hline YG-28 & Medium & 107,5 & 10,6 & 26,6 & $1-3$ & 11 & 30,4 & 47,5 & 9,5 & Light \\
\hline YG-28/2 & Medium & 91,0 & 7,3 & 29,6 & 1 & 10,6 & 33,7 & 104,0 & 6,0 & Medium \\
\hline YG-29 & Medium & 80,5 & 8,2 & 27,3 & $1-3$ & 10,5 & 29,65 & 81,0 & 11,0 & Light \\
\hline YG-30 & Medium & 77,0 & 7 & 27,6 & $1-3$ & 12,5 & 33,6 & 176,0 & 5,5 & Medium \\
\hline YG-31 & Weak & 86,0 & 8,3 & 24,3 & $1-3$ & 15,3 & 31,45 & 143,0 & 8,0 & Light \\
\hline YG-31/2 & Medium & 83,5 & 8,5 & 27,3 & $1-3$ & 11,1 & 30,4 & 37,3 & 12,0 & Light \\
\hline YG-32 & Medium & 96,5 & 10,0 & 26,3 & $1-3$ & 13,6 & 34,8 & 81,0 & 9,5 & Light \\
\hline YG-32/2 & Medium & 98,0 & 8,2 & 29,0 & $1-3$ & 21 & 25,2 & 184,0 & 6,5 & Light \\
\hline YG-33 & Medium & 98,5 & 9,0 & 17,0 & 1 & 8,5 & 27,7 & 36,3 & 7,5 & Light \\
\hline YG-33/2 & Medium & 80,2 & 7,5 & 27,3 & $1-3$ & 20,1 & 39,5 & 166,0 & 8,5 & Medium \\
\hline YG-34 & Medium & 73,5 & 8,4 & 30,0 & $1-3$ & 21,8 & 34,9 & 245,0 & 8,5 & Medium \\
\hline YG-35 & Medium & 95,0 & 8,5 & 27,6 & 1 & 15,8 & 34,7 & 270,0 & 10,0 & Light \\
\hline YG-36 & Medium & 106,5 & 10,2 & 28,3 & $1-3$ & 16,8 & 35,9 & 135,5 & 8,0 & Medium \\
\hline YG-36/2 & Medium & 89,0 & 7,5 & 28,0 & $1-3$ & 15,1 & 35,6 & 56,0 & 8,5 & Light \\
\hline YG-37 & Medium & 101,0 & 8,5 & 26,0 & $1-3$ & 22,6 & 38,2 & 381,0 & 11,0 & Medium \\
\hline YG-38 & Strong & 130,2 & 11,3 & 27,6 & 1 & 32,5 & 42,05 & 585,0 & 9,5 & Intense \\
\hline
\end{tabular}

In this study, 20 morphologic descriptors which were derived from UPOV by Gozen (2008) used for the definition of the phylogenetic relationships among the 90 Turkish cucumber landraces. Obtained morphological data was used to draw the dendrogram (Figure 2) generated by the UPGMA method. 


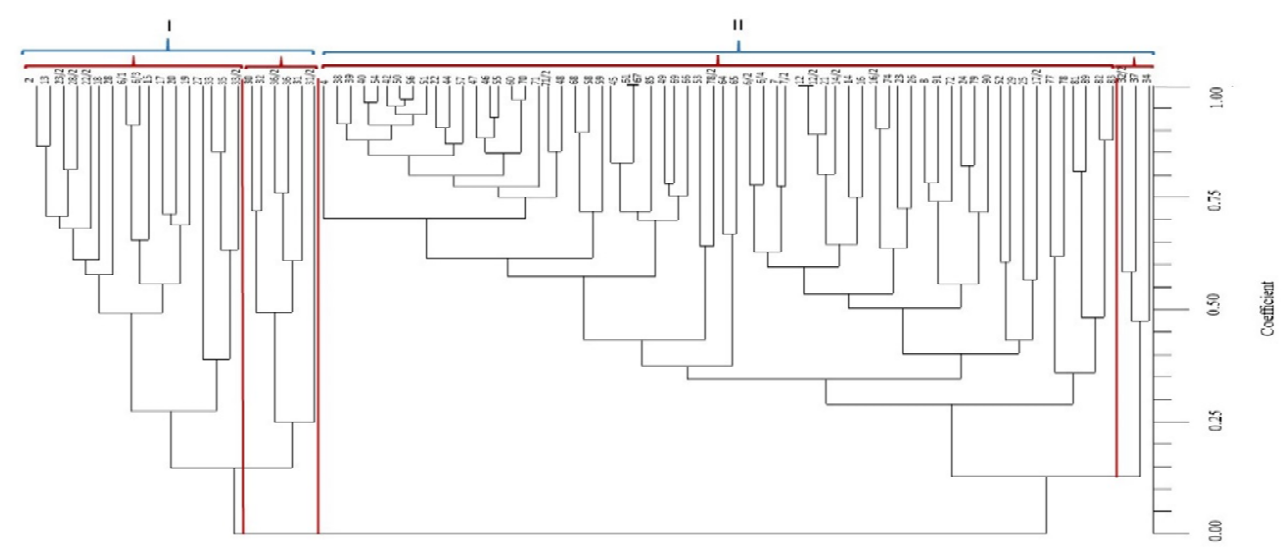

Figure 2. UPGMA dendrogram showing phylogenetic relationships of local cucumber genotypes using morphological data

According to the dendrogram presented in Figure 2, two major and four subgroups were revealed with the similarity rate of $\% 25$. Although the most distant genotypes were determined YG-2 and YG-34 gathered from Canakkale, the most similar ones were YG-61 from Mersin, YG-67 from Antalya (Figure 3). Thus, the fact that, the origins of genotypes were close or far apart did not affect their morphological similarity in the research.
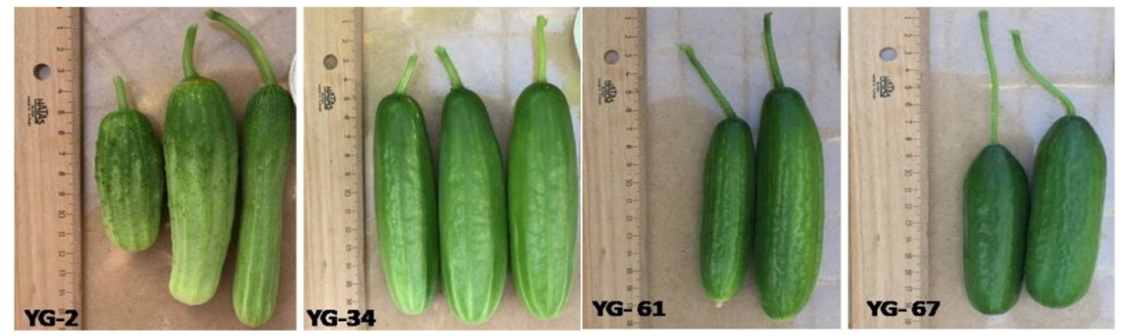

Figure 3. The most distant and similar genotypes according to UPGMA dendrogram which was drawn using morphological descriptors

Determination of the genetic diversity of a gene pool is crucial for the success of the breeding programs. Because they are more reliable and consistent, using neutral markers is essential in such studies (Al-Rawahi $e t$ al., 2011). Therefore, morphological and molecular markers were preferred in the current study and the discrimination power of each polymorphic marker (estimated by the polymorphism information content) ranged from 0.15 to 0.99 with an average of 0.73 . Dice's similarity coefficient ranged from 0.00 to 1.00 . The dendrogram provided from the molecular data was given in Figure 4. Accordingly, 90 genotypes used in the study divided into 2 main clusters which were subdivided into themselves. Genetic diversity and relationships of the genotypes were revealed using 20 sequence-related amplified polymorphism (SRAP) marker combinations.

Genotypes namely YG-15 (from Rize), 6/1, 6/2, 6/3, 6/4 (from Edirne), 33 (from Nevsehir), 35 (from Istanbul) and 83 (from Kilis) in the cluster $\mathrm{C}$ created a very different group than the other 82 genotypes (Figure 4). Among the genotypes included in cluster C; YG-15, YG-33, YG-35 were understood to be Cucumis melo var. flexuosus and YG-6/1-2-3-4 and YG-83 were thought as wild cucumber genotypes (Figure 5). 


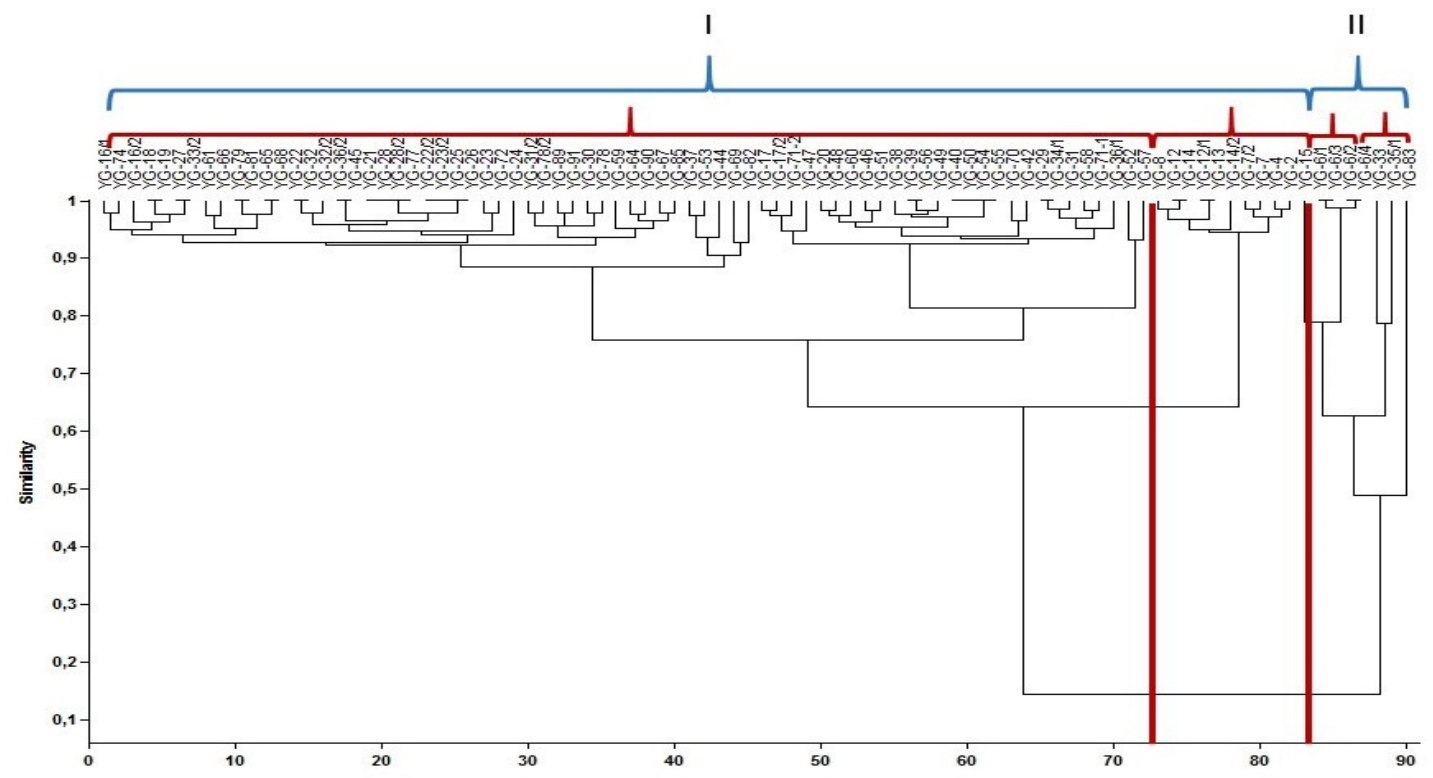

Figure 4. UPGMA dendrogram showing similarity of local cucumber genotypes using molecular data

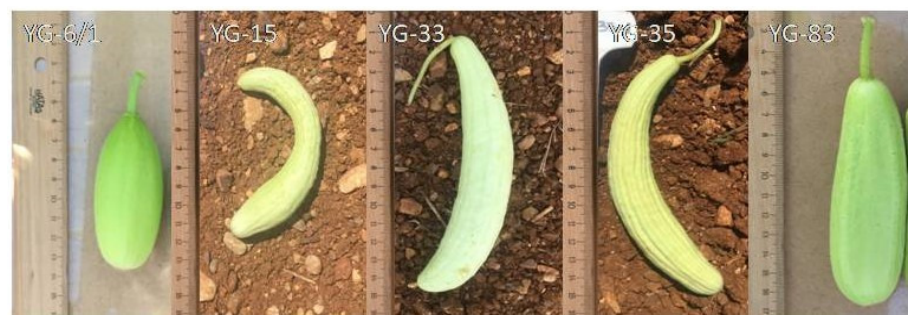

Figure 5. Genotypes in cluster $\mathrm{C}$ in terms of the SRAP analysis

In addition to this, YG-15 and YG-83 were defined as the most distant genotypes of the Cluster C. In terms of the morphologic data, while the YG-6/1-3, YG-15, YG-33, and YG-35 were in the group 1, YG-6/24 and YG-83 were found in group 2. The phenomenon of hybrid vigour occurring from the crosses between genetically distinct genotypes forms a prominent meaning of crop improvement (Tiwari, 2015). Therefore, morphologically similar and molecularly distinct genotypes could be used as parent lines in the further cucumber breeding studies.

According to the dendrogram presented in Figure 4, there were 37 genotypes in cluster B, remaining 45 genotypes were found in cluster A. According to Dice similarity and distance indexes group A consisted of genetically the most similar genotypes with the smallest similarity value of 0.88 . Mliki et al. (2003) studied the genetic diversity of 26 African cucumber accessions. Their data suggest that some genotypes from Egypt have unique genetic diversity and this germplasm has the potential for broadening the genetic base of cucumber breeding studies. Gaikwad (2011) reported that genotypic variation was slightly low as compared to phenotypic variation in cucumber. The highest genotypic, as well as phenotypic variation was observed on fruit length, fruit number, and fruit weight. With the current study, it was cleared that Turkey has reasonable genetic diversity in terms of cucumber and the 20 SRAP primer combinations established here could be used for germplasm identification in further classification studies. Today management of biodiversity for sustainable food has become a significant subject. Landraces of every country represent a major source of diversity because of the adaptation capability to the habitats of origin. These resources could be used in the plant breeding programs for the constitution of more resilient and qualitatively improved varieties (Parisi et al., 2017). 
In Table 5, used 20 SRAP primer combinations and their number of total alleles, polymorphic alleles, polymorphism rates (\%), and polymorphism information content (PIC) values were presented. Considering these, the most allelic primer combination was found as me11-em9 with the total 12 alleles. The least allelic primer combinations were determinate as me12-em 10 and me6-em 16 with the 5 alleles. Polymorphism rate (\%) was ranked between \%16- \%100.

Table 5. Total alleles, polymorphic alleles, polymorphism rate (\%), and PIC values of used SRAP primer combinations

\begin{tabular}{|c|c|c|c|c|}
\hline Primers & Total alleles & Polymorphic alleles & Polymorphism rate (\%) & PIC* \\
\hline me1-em4 & 9 & 6 & 66.67 & 0.69 \\
\hline me1-em11 & 6 & 1 & 16.67 & 0.15 \\
\hline me2-em10 & 8 & 4 & 50.00 & 0.48 \\
\hline me2-em7 & 6 & 1 & 16.67 & 0.99 \\
\hline me3-em7 & 8 & 3 & 37.50 & 0.43 \\
\hline me3-em15 & 9 & 7 & 77.78 & 0.98 \\
\hline me4-em13 & 7 & 3 & 62.86 & 0.85 \\
\hline me4-em14 & 11 & 7 & 22.22 & 0.99 \\
\hline me5-em6 & 9 & 2 & 45.45 & 0.99 \\
\hline me5-em13 & 11 & 5 & 80.00 & 0.82 \\
\hline me6-em16 & 5 & 4 & 50.00 & 0.99 \\
\hline me8-em16 & 8 & 4 & 33.33 & 0.99 \\
\hline me8-em11 & 6 & 2 & 100.00 & 0.90 \\
\hline me9-em2 & 6 & 6 & 55.56 & 0.99 \\
\hline me9-em11 & 9 & 5 & 50.00 & 0.82 \\
\hline me10-em9 & 6 & 3 & 58.33 & 0.38 \\
\hline me11-em9 & 12 & 7 & 25.00 & 0.59 \\
\hline me12-em2 & 8 & 2 & 80.00 & 0.52 \\
\hline me12-em10 & 5 & 4 & 42.86 & 0.45 \\
\hline me15-em5 & 7 & 3 & - & 0.63 \\
\hline Total & 156 & 79 & 50.73 & - \\
\hline Mean & 7.8 & 3.9 & & 0.73 \\
\hline Polym & & & & \\
\hline
\end{tabular}

${ }^{*}$ Polymorphism information content

\section{Conclusions}

With this project, 90 of the local cucumber genotypes grown in different regions of Turkey were collected and morphologically characterized considering the 20 UPOV descriptors. Later, genetic similarity of the genotypes was identified between the $0.00-1.00$ using 20 SRAP primer combinations showing polymorphism. Used marker systems also allowed the efficient management of the gene pool by eliminating redundant accessions. It was cleared that Turkey has reasonable diversity regarding cucumber. It was also understood that the obtained gene pool has a potential for broadening the genetic base of cucumber for further breeding studies. According to the clusters achieved in the study, morphologically similar and molecularly distinct genotypes could be used as parent lines in the further cucumber breeding studies.

\section{Authors' Contributions}

LK and VG collected the cucumber landraces. AY did molecular studies in the laboratory. EC did all the field works and morphological observations, measurements etc., in addition she evaluated the data and wrote the manuscript. All authors read and approved this final version of the manuscript. 


\section{Acknowledgements}

The authors of the study would like to thank to Dr. H. Filiz Boyaci for the valuable technical advices. This manuscript produced from the project conducted between the years of 2016-2019 titled "Morphological and Molecular Characterization of Some Local Cucumber (Cucumis sativus L.) Genotypes” supported by the General Directorate of Agricultural Research and Policies. This research did not receive any specific grant from funding agencies in the public, commercial, or not-for-profit sectors.

\section{Conflict of Interests}

The authors declare that there are no conflicts of interest related to this article.

\section{References}

Agarwal M, Shrivastava N, Padh H (2008). Advances in molecular marker techniques and their applications in plant sciences. Plant Cell Reports 27(4):617-631. https://doi.org/10.1007/s00299-008-0507-z

Ahmed M, Abdul H, Zarqa A (2004). Growth and yield performance of six cucumber (Cucumis sativus L.) cultivars under agro-climatic conditions of Rawalakot, Azad Jammu and Kashmir. International Journal of Agriculture and Biology 6:396-399.

Al-Rawahi MF, Al-Said A, Khan IA, Al-Khanjary S (2011). Diversity of cucumber accessions in Oman. International Journal of Agriculture and Biology 13:505-510.

Aydemir I (2009). Determination of genetic diversity in cucumber (Cucumis sativus L.) germplasms. MSc thesis, İzmir Institute of Technology, pp 40.

Bartaula S, Adhikari A, Panthi U, Karki P, Timalsena K (2019). Genetic variability, heritability and genetic advance in cucumber (Cucumis sativus L.). Journal of Agriculture and Natural Resources 2(1):215-222.

Bisognin DA (2002). Origin and evolution of cultivated cucurbits. Ciencia Rural 32:715-723.

Dice LR (1945). Measures of the amount of ecologic association between species. Ecology 26:297-302.

Doyle JJ, Doyle, JL (1990). A rapid DNA isolation procedure for small quantities of fresh leaf tissue. Phytochemical Bullettin 19(1):11-15.

Fares K, Guasmi F, Touil L, Triki T, Ferchini A (2009). Genetic diversity of pistachio tree using inter simple sequence repeat markers (ISSR) supported by morphological and chemical markers. Biotechnology 8(1):24-34. https://scialert.net/abstract/?doi=biotech.2009.24.34

Ferriol M, Pico B, Nuez F (2003). Genetic diversity of some accessions of Cucubita maxima from Spain using RAPD and SRAP markers. Genetic Resources and Crop Evolution 50(3):227-238.

Ferriol M, Pico B, de Cordova PF, Nuez F (2004). Molecular diversity of a germplasm collection of squash (Cucurbita moschata) determined by SRAP and AFLP markers. Crop Science 44:653-664.

Gaikwad AG, Musmade AM, Dhumal SS, Sonawane HG (2011). Variability studies in cucumber (Cucumis sativus L.). Ecology Environment and Conservation 17(4):799-802.

Gozen V (2008). Morphologic characterization in hybrid greenhouse cucumber breeding and determination of appropriate hybrid combinations and hybrid seed quality. Phd thesis, Ankara pp 185.

Gulsen O, Mutlu N (2005). Bitki biliminde kullanılan genetic markırlar ve kullanım alanları. Alatarım 4(2):27-37.

Gostimsky SA, Kokaeva ZG, Konovalov FA (2005). Studying plant genome variation using molecular markers. Russian Journal of Genetics 41(4):378-388.

Kennard WC, Havey MJ (1995). Quantitative trait analysis of fruit quality in cucumber: QTL detection, confirmation and comparison with mating-design variation. Theoretical and Applied Genetics 91:53-61.

Kumar D, Kumar S, Singh J, Narender, Rashmi, Vashistha BD, Singh N (2010). Free radical scavenging and analgesic activities of Cucumis sativus L. fruit extract. Journal of Young Pharmacists 2:365-368. https://doi.org/10.4103/0975-1483.71627 
Mliki A, Staub JE, Zhangyong S, Ghorbel A (2003). Genetic diversity in African cucumber (Cucumis sativus L.) provides potential for germplasm enhancement. Genetic Resources and Crop Evolution 50(5):461-468.

Normohamadi S, Solouki M, Heidari F (2017). Diversity in cucumber genotypes based on morphological traits and SSR molecular markers. Biosciences Biotechnology Research Asia 14(2):775-782. http://dx.doi.org/10.13005/bbra/2507

Parisi M, Di Dato F, Ricci S, Mennella G, Cardi T, Tripodi P (2017). A multi-trait characterization of the 'Friariello' landrace: a Mediterranean resource for sweet pepper breeding. Plant Genetic Resources 15(2):165-176. https://doi.org/10.1017/S1479262115000490

Rao VR, Hodgkin T (2002). Genetic diversity and conservation and utilization of plant genetic resources. Plant Cell, Tissue and Organ Culture 68:1-19.

Rohlf FJ (1998). NTSYS-PC: Numerical taxonomy and multivariate analysis system. Release 2.20j. Exeter Software, Setauket, N.Y.

Sebastian P, Schaefer H, Telford IR, Renner SS (2010). Cucumber and melon have numerous wild relatives in Asia and Australia, and the sister species of melon is from Australia. Proceedings of the National Academy of Sciences 107(32):14269-14273

Smith JSC, Chin ECL, Shu H, Smith OS, Wall SJ, Senior ML, ... Ziegle J (1997). An evaluation of the utility of SSR loci as molecular markers in maize (Zea mays L.): comparisons with data from RFLPS and pedigree. Theoretical and Applied Genetics 95:163-173.

Staub JE, Serquen FC, Horejsi T, Chen JF (1999). Genetic diversity in cucumber (Cucumis sativus L.): IV. An evaluation of Chinese germplasm. Genetic Resources and Crop Evolution 46(3):297-310.

Tiwari R (2015). Molecular characterization and genetical studies in cucumber (Cucumis sativus L.) (Doctoral dissertation, Ph.D. (Agri.) Thesis, Govind Ballabh Pant University of Agriculture and Technology, Pantnagar, Uttarakhand, India, pp 129.

Wehner TC, Robinson RW (1991). A brief history of the development of cucumber cultivars in the US. Cucurbit Genetics Cooperative 14:1-4.

Wehner TC, Guner N (2002). Growth stage, flowering pattern, yield, and harvest date prediction of four types of cucumber tested at 10 planting dates. In: XXVI International Horticultural Congress: Advances in Vegetable Breeding 637:223-230.

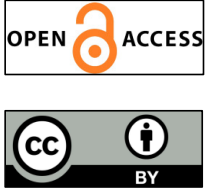

The journal offers free, immediate, and unrestricted access to peer-reviewed research and scholarly work. Users are allowed to read, download, copy, distribute, print, search, or link to the full texts of the articles, or use them for any other lawful purpose, without asking prior permission from the publisher or the author.

License - Articles published in Notulae Botanicae Horti Agrobotanici Cluj-Napoca are Open-Access, distributed under the terms and conditions of the Creative Commons Attribution (CC BY 4.0) License. (c) Articles by the authors; UASVM, Cluj-Napoca, Romania. The journal allows the author(s) to hold the copyright/to retain publishing rights without restriction. 